\title{
COL4A1 Mutation in a Pediatric Patient Presenting with Post-Ictal Hemiparesis
}

\author{
Marian Leung, Evan Lewis, Peter Humphreys, Elka Miller, Michael Geraghty, Matthew Lines, \\ Erick Sell
}

A six-year-old girl presented with a prolonged left sided paresis and acute complete right hemispheric injury of uncertain mechanism following an episode of convulsive status epilepticus. She was born at full term after an uneventful pregnancy. At day two of life, she had three consecutive seizures and magnetic resonance imaging (MRI) revealed an intraparenchymal small bleed in the right frontal lobe. Full coagulation workup was done which was negative. She was successfully treated with phenobarbital. Follow-up MRI at seven months revealed right frontal porencephaly and volume loss of the right hemispheric subcortical white matter, basal ganglia, thalami, cerebral peduncle and corpus callosum. Abnormal signal in the right cerebellar hemisphere was also identified. At 12 months-of-age, brain computed tomogram angiography indicated no evidence of arteriovenous malformations. Electroencephalogram (EEG) at 18 months-of-age showed a mildly disorganized background for age and asymmetry throughout the recording with lower amplitudes in the right hemisphere. Neurological examinations were consistently abnormal with increased reflexes on the left and a tendency to favor her right side. At two years-of-age, repeat MRI with MRA was unchanged and there was no evidence of vasculopathy (Figure1A). She remained seizure-free. Pheno-barbital was discontinued and she was discharged from follow-up.

The child then presented to our emergency department at six years-of-age with a generalized tonic-clonic seizure that lasted 35 minutes and was aborted with intravenous lorazepam and dilantin. Seizure onset was not witnessed, however several hours earlier she had awoken from sleep with a headache, sore throat, malaise and complaints of being warm. Physical examination after recovery from her seizure revealed normal vitals and she was afebrile. She was found to have dysarthria, a left homonymous hemianopsia, a left dense hemiparesis, and left sided upper motor neuron signs including a left extensor plantar response. Blood work including cultures, extractable nuclear antigen, antinuclear antibodies, and a coagulation profile were all normal. A cardiology assessment including electrocardiogram and echocardiogram were normal. Brain MRI demonstrated diffuse right cortical and subcortical edema with associated reduced diffusivity of water motion in the right hemisphere, involving areas supplied by both the anterior and posterior circulations (Figure 2). The MRA studies indicated no evidence of a vascular malformation or vessel abnormalities. Previously described structural abnormalities were unchanged. An EEG revealed abnormal background and right-sided diffusely low
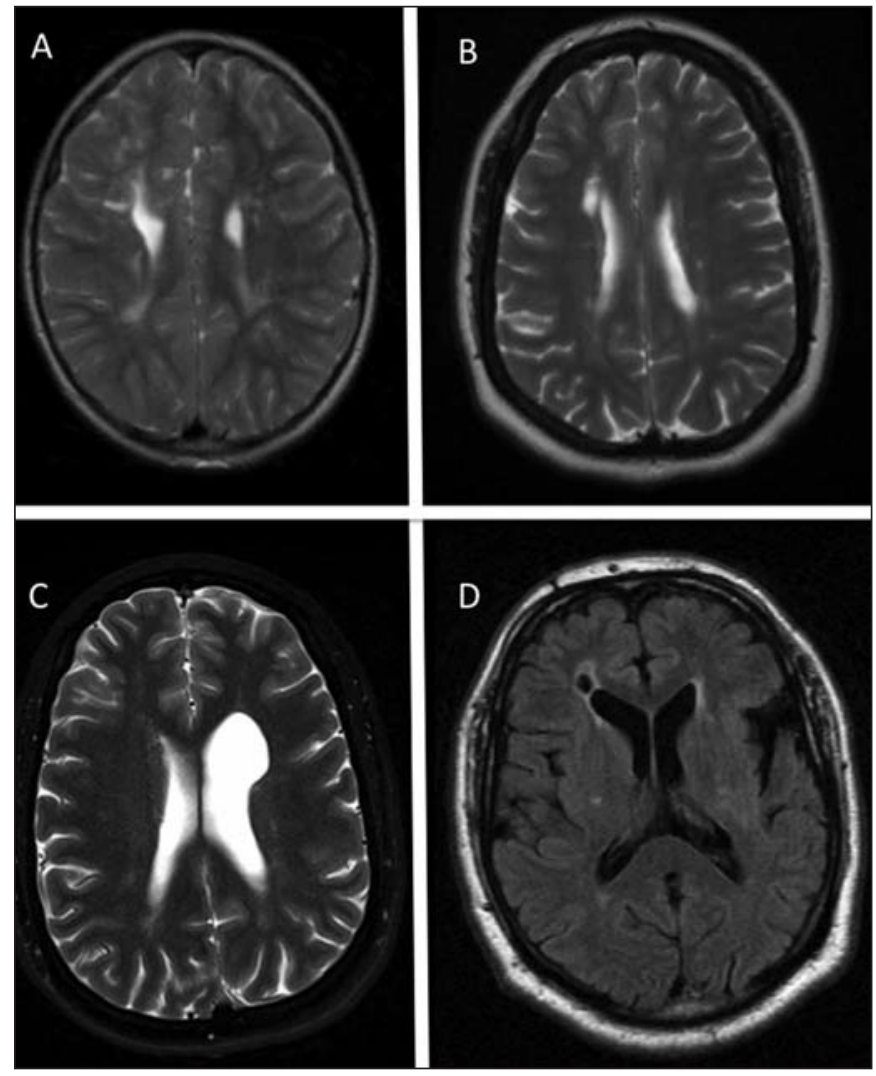

Figure 1: MRI of patient, mother, maternal aunt \& maternal grandfather. (A) patient MRI at two years of age, T2WI; (B) MRI of patient's mother at thirty years of age, T2W1; (C) MRI of patient's aunt at twenty-four years of age, T2WI; (D) MRI of patient's grandfather at fifty-six years of age, T2 FLAIR. All subjects have similar encephalomalacia around the right or left superior frontal horn and signal changes in the periventricular regions.

From the Faculty of Medicine (MLe), University of Ottawa; Department of Neurology (ECL, PH, ES), Department of Radiology (EM), Department of Genetics (MG, MLi), Children's Hospital of Eastern Ontario, Ottawa, Ontario, Canada.

Received January 4, 2012. Final Revisions Submitted March 21, 2012. Correspondence to: Marian Leung, University of Ottawa - Faculty of Medicine, 451 Smyth Rd., Ottawa, Ontario, K1H 8M5, Canada. Email: mleun097@uottawa.ca. 

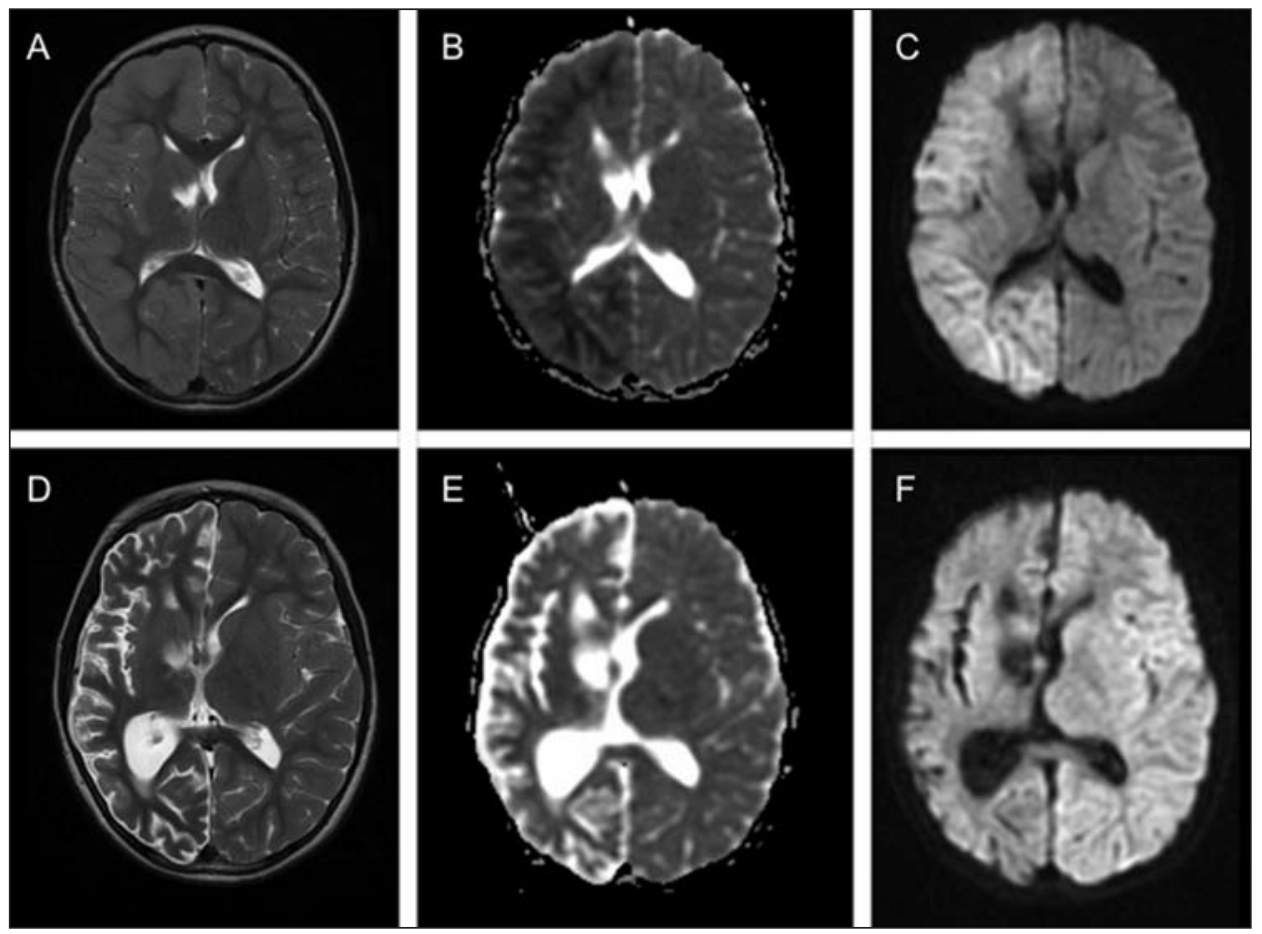

Figure 2: MRI of patient. (A-C) at time of presentation following status epilepticus; $(D-F)$ one month after presentation. (A and D) T2WI; ( $B$ and E) Apparent Diffusion Coefficient; ( $C$ and F) Diffusion Weighted Imaging. Comparison of patient's MRIs reveal almost complete diffusion restriction of the right hemisphere at presentation $(B$ and $C)$ and one month later significant volume loss affecting the same territory $(D-F)$.

voltage with an admixture of delta and theta waves most pronounced over the frontal leads. There was no evidence of epileptiform activity. She was started on carbamazepine.

Follow-up MRI a month after the acute event revealed marked loss of volume of the entire right cerebrum (Figure 2). No vascular abnormalities were visualized. At this time, she was interactive and able to talk throughout the exam. There was persistence of a left homonymous hemianopsia. The left arm revealed increased tone and fine motor control difficulties. She had brisk reflexes in the left upper and lower extremities and a left extensor plantar response. At three months of follow-up, she remained seizure-free with some recovery of fine motor abilities in her left hand. Neuropsychological testing showed significant deficits in language, processing speed, attention, memory, visual spatial ability and academic achievement. She had two more EEGs in follow-up that continued to show a slow and poorly organized background for age. The right-sided asymmetry became much more apparent following her episode of status epliepticus. Frequency on the right side was formed from a mixture of delta and theta waves and amplitudes were consistently low with average voltages less than $25 \mu \mathrm{V}$.

Family history revealed several members affected by unexplained neurologic disease (Figure 3). The imaging studies of the maternal aunt (Figure 1C) showed similar findings to our patient except the left hemisphere was affected instead of the right. This led to genetic testing for the COL4A1 mutation. Our patient, the mother, the maternal aunt, the maternal grandfather and a first cousin were all determined to be heterozygous for a missense mutation in COL4A1 (c.4031G>C; p.Gly1344Ala).

After these results were obtained, MRI imaging of our patient's mother was conducted and past imaging of the maternal grandfather was reviewed.The MRI findings were similar to those found in our patient and the maternal aunt (Figures 1A-D). No imaging of the first cousin was undertaken as she has remained asymptomatic.

\section{Discussion}

Here we describe a patient with right hemispheric volume loss after an episode of status epilepticus in the context of a COL4A1 mutation. This is the first report of this association thereby broadening the clinical and radiological phenotype of COL4A1 related diseases. This case illustrates that COL4A1 may predispose patients to significant neurological consequences in the context of status epilepticus.

Mutations in the COL4A1 gene, encoding type IV procollagen, appear to cause generalized vascular disease most often affecting the small vessels of the brain. This mutation can produce a wide variety of clinical phenotypes such as porencephaly, cerebral small vessel disease, infantile hemiparesis, perinatal stroke, leukoencephalopathy, deep intracerebral hemorrhage, and retinal arteriolar tortuosity. ${ }^{1-3}$ There have also been reports of COL4A1 mutations and diffuse leukoencephalopathy with Axenfeld-Rieger type ocular anterior chamber malformations and HANAC - hereditary angiopathy, 


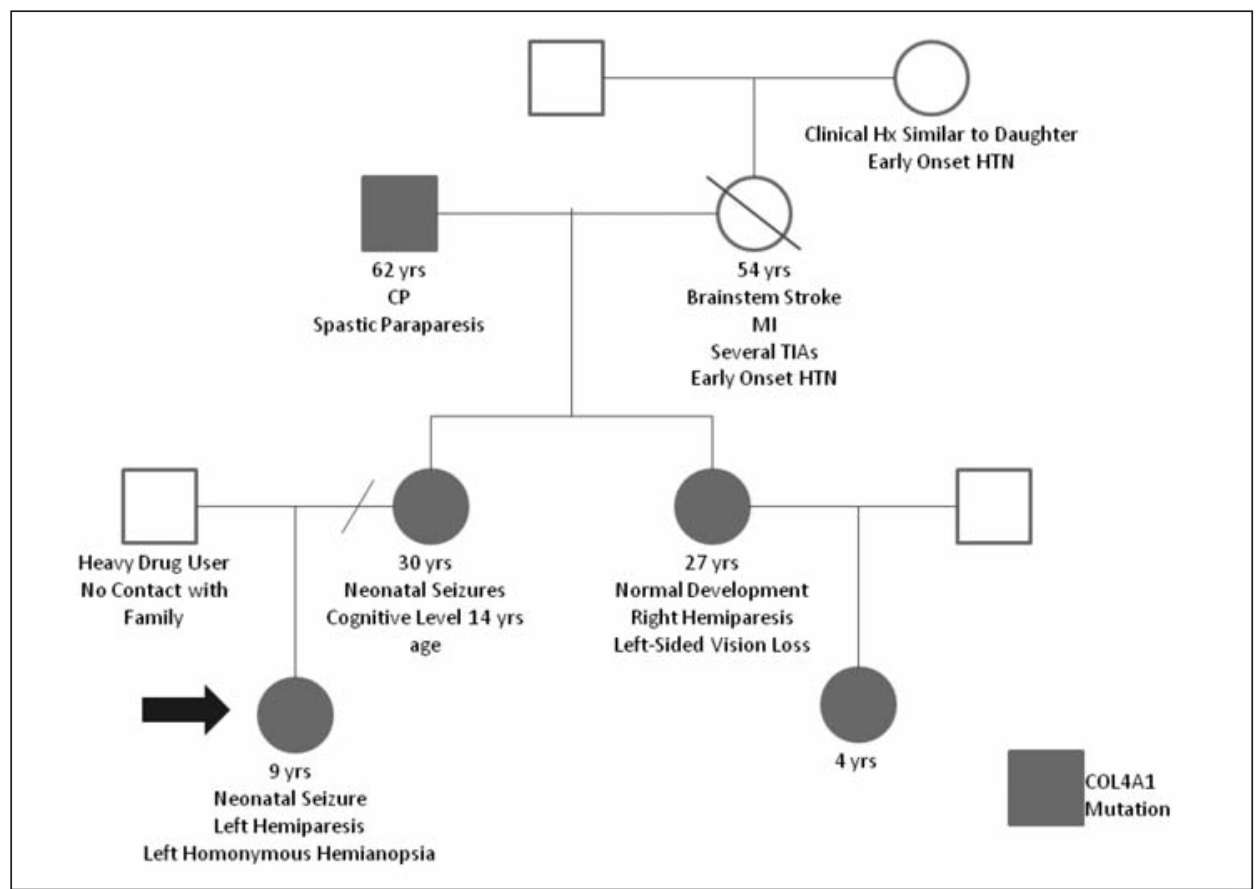

Figure 3: Family tree of patient (2011). Family tree of the proband with those affected by the COLAA1 mutation represented in purple.

Table 1: Other monogenic causes of small vessel diseases of the brain. [1]

\begin{tabular}{|c|c|c|c|c|}
\hline Disorder & Inheritance Pattern & Average Onset Age & Clinical Characteristics & Gene \\
\hline COL4A1-related disorder & $\begin{array}{l}\text { Autosomal } \\
\text { dominant }\end{array}$ & $14-49$ & $\begin{array}{l}\text { Infantile hemiparesis } \\
\text { Congenital porencephaly } \\
\text { Intracerebral hemorrhages } \\
\text { Seizures }\end{array}$ & COL4A1 \\
\hline COL4A2-related disorder & $\begin{array}{l}\text { Autosomal } \\
\text { dominant }\end{array}$ & & $\begin{array}{l}\text { Congenital porencephaly } \\
\text { White matter lesions } \\
\text { Cerebellar and optic nerve hypoplasia }\end{array}$ & COL4A2 \\
\hline $\begin{array}{l}\text { CADASIL } \\
\text { (Cerebral autosomal } \\
\text { dominant arteriopathy with } \\
\text { subcortical infarcts and } \\
\text { leukoencephalopathy) }\end{array}$ & $\begin{array}{l}\text { Autosomal } \\
\text { dominant }\end{array}$ & $40-50$ & $\begin{array}{l}\text { Migraines + aura } \\
\text { Ischemic strokes/TIA } \\
\text { Progressive cognitive dysfunction } \\
\text { Psychiatric mood disorders }\end{array}$ & NOTCH3 \\
\hline $\begin{array}{l}\text { CARASIL } \\
\text { (Cerebral autosomal } \\
\text { recessive arteriopathy with } \\
\text { subcortical infarcts and } \\
\text { leukoencephalopathy) }\end{array}$ & $\begin{array}{l}\text { Autosomal } \\
\text { recessive }\end{array}$ & $20-30$ & $\begin{array}{l}\text { Ischemic strokes } \\
\text { Progressive dementia } \\
\text { Alopecia } \\
\text { Lumbar intervertebral disc herniations } \\
\text { Osseous deformities }\end{array}$ & HTRA1 \\
\hline $\begin{array}{l}\text { RVCL } \\
\text { (Retinal vasculopathy with } \\
\text { cerebral leukodystrophy) }\end{array}$ & $\begin{array}{l}\text { Autosomal } \\
\text { dominant }\end{array}$ & $30-50$ & $\begin{array}{l}\text { Ischemic strokes } \\
\text { Pseudotumour } \\
\text { Seizures } \\
\text { Motor and sensory deficits } \\
\text { Renal disease }\end{array}$ & TREX1 \\
\hline $\begin{array}{l}\text { Fabry Disease } \\
\text { (Alpha-galactosidase A } \\
\text { deficiency) }\end{array}$ & $\mathrm{X}$-linked recessive & $6-10$ & $\begin{array}{l}\text { Acroparesthesia } \\
\text { Angiokeratoma } \\
\text { Hypohidrosis } \\
\text { GI difficulties } \\
\text { Renal insufficiency } \\
\text { Cardiomyopathy } \\
\end{array}$ & GLA \\
\hline $\begin{array}{l}\text { MELAS } \\
\text { (Mitochondrial } \\
\text { encephalomyopathy, lactic } \\
\text { acidosis, and stroke-like } \\
\text { episodes) }\end{array}$ & $\begin{array}{l}\text { Maternal } \\
\text { inheritance }\end{array}$ & $4-15$ & $\begin{array}{l}\text { Vomiting } \\
\text { Headaches } \\
\text { Seizures } \\
\text { Hearing loss } \\
\text { Muscle weakness/pain } \\
\text { Learning disability } \\
\text { Diabetes mellitus } \\
\text { Cardiac disease }\end{array}$ & $\begin{array}{l}\text { MT-TL1 or } \\
\text { other } \\
\text { mtDNA }\end{array}$ \\
\hline
\end{tabular}

* Table not exhaustive. Various other causes not listed. 
nephropathy, aneurysms, and muscle cramps. ${ }^{1}$ COL4A1 mutations add to the list of other known monogenic causes of small vessel brain disease (Table).

Our patient's COL4A1 mutation (p.Gly1344Ala) occurs in a highly conserved glycine residue in a Gly-X-X motif of the triple helical domain of the protein. This amino acid change can therefore, be predicted to adversely affect formation of the collagen triple helix. ${ }^{1}$ In mice, electron microscopy studies have shown that disruption of Col4a1 compromises the structural integrity of the cerebral vascular basement membrane, altering its density and thickness. ${ }^{2}$ To date, there have been no pathological studies of cerebral vessels in human patients with COL4A1 mutation. However, electron micrographs of skin capillaries in a patient with a different but comparable missense mutation (p.Gly1236Arg) of COL4A1 showed focal interruptions or increased thickness of the basement membrane. ${ }^{3}$ This could be the pathologic mechanism in our patient as well.

Our patient exhibited features described in previous COL4A1 mutation reports such as: seizures, infantile hemiparesis, ischemic lacunar strokes, mild cognitive delay, and white matter hyperintensities on imaging., ${ }^{1,3}$ Porencephaly is also a welldocumented clinical effect of COL4A 1 which was seen in our patient, her aunt and her mother. In our patient, the weakened collagen defective vessels may have been more susceptible to rupture from an unknown prenatal or perinatal stress leading to the intraparenchymal hematoma seen on her neonatal MRI and subsequent evolution to porencephly in the same location. Our patient also showed right hemispheric thinning of the subcortical white matter and atrophy of the right basal ganglia, thalamus, cerebral peduncle, and corpus callosum. These extensive rightsided abnormalities do not conform to a single vascular territory and the biologic mechanism to explain these static unilateral findings in the context of a COL4A 1 mutation is not as clear.

It has been well documented that prolonged seizures can lead to metabolic and hypoxic ischemic brain injury and subsequent neuronal loss. ${ }^{4}$ We speculate that our patient's COL4A1 defect may have predisposed her to more significant consequences as a result of her prolonged seizure. This lead to the development of adverse neurological outcomes that were worse than would have been expected in an otherwise normal patient. A study looking at the outcome of convulsive status epilepticus in children found that the risk of developing focal neurological deficits, cognitive impairment, and behavioural problems was dependant on the etiology of the seizure. Twenty percent of the children who developed new neurological sequelae had a remote symptomatic etiology, which was defined as "status epilepticus in the absence of an identified acute insult but with a history of a CNS insult."5 This evidence supports our hypothesis that the abnormality of the basement membranes in our patient's blood vessels as a consequence of the COL4A1 mutation may have contributed to the diffuse neuronal loss seen in our patient after her prolonged seizure. This underlying abnormality likely exacerbated the ischemic insult suffered by the prolonged status epilepticus resulting in cytotoxic edema and neuronal cell death beyond what we would expect to find in a patient suffering a prolonged seizure with normal brain physiology. That the damage provoked by the period of status epilepticus was confined to the previously abnormal hemisphere may reflect the possibility that the early (unwitnessed) portion of the seizure involved only the hemisphere containing the epileptogenic focus. Alternatively, the combination of a COL4A1 mutation and previous structural damage may have rendered the right cerebral hemisphere more vulnerable to the effects of a prolonged generalized seizure.

There is wide variability in the phenotypic presentation of COL4A1-related diseases making the identification of this condition more challenging. A recent review, however, described emerging patterns amongst the known human phenotypes suggesting unique clinical features of COL4A1 mutations compared to other monogenic causes of small vessel disease. ${ }^{2}$ These features include: intracerebral hemorrhage in children and young adults but predominating in the neonatal period, diffuse leukoencephalopathy secondary to diffuse small vessel disease, retinal arteriolar tortuosities, retinal hemorrhages, intracranial aneurysms, porencephaly, infantile hemiparesis, muscle cramps, renal involvement, secondary glaucoma and muscle cramps. Patients with a confirmed COL4A1 mutation or suspected on clinical grounds, should undergo a thorough work-up to evaluate for extra-neurological manifestations as described above. Preventative measures should also be taken for at-risk pregnancies and patients should be counseled on avoidance of risk factors for hemorrhagic stroke.

This case provides an expansion of the COL4A1 phenotype, proposing that COL4A1 mutations should be considered in patients with a history of porencephaly who present with seizures of unknown etiology and have accompanying clinical and/or radiological evidence of postictal manifestations that are out of keeping with their initial presentations. In addition, this case may suggest that in patients with seizures and known COL4A1 mutations, lowering the threshold to initiate anticonvulsant treatment might be beneficial with more restrictive indications for discontinuing anti-epileptic medications. Further research is needed to explore the potential role for COL4A1 mutations in extensive ischemic damage associated with epileptic seizures.

\section{REFERENCES}

1. Yamamoto Y, Craggs L, Baumann M, Kalimo H, Kalaria RN. Molecular genetics and pathology of hereditary small vessel diseases of the brain. Neuropathol Appl Neurobiol. 2011;37: 94-113.

2. Vahedi K, Alamowitch S. Clinical spectrum of type IV collagen (COL4A1) mutations: a novel genetic multisystem disease. Curr Opin Neurol. 2011;24:63-8.

3. van der Knaap MS, Smit LME, Barkhof F, et al. Neonatal porencephaly and adult stroke related to mutations in collagen IVA1. Ann Neurol. 2006;59:504-11.

4. Milligan TA, Zamani A, Bromfield E. Frequency and patterns of MRI abnormalities due to status epilepticus. Seizure. 2008;18: 104-8.

5. Novorol CL, Chin RFM, Scott RC. Outcome of convulsive status epilepticus: a review. Arch Dis Child. 2007;92(11):948-51. 